\title{
Application of Neural Network in the Evaluation of Equipment Maintenance Quality
}

\author{
Zhang Meng, Xu Peng-fei, Liu Jun-bang, Jiang Wei and Wang Shuai \\ Wuhan Mechanical Technology College \\ Wuhan, Hubei Province, China \\ jw007305@yahoo.com.cn
}

\begin{abstract}
Keywords: BP Neural Network; Neural Network Toolbox; the evaluation model of maintenance quality
\end{abstract}

\begin{abstract}
Using neural network BP algorithm and the neural network toolbox of MATLAB, this paper presents a new the evaluation model of equipment maintenance quality and uses an example to validate the evaluation model of equipment maintenance quality. At last it is proved that the effect of the model is perfect.
\end{abstract}

\section{Introduction}

The equipment maintenance is the important factor to keep, resume and improve the battle effectiveness. The problem that how to strengthen the maintenance quality control and how to improve the maintenance quality level and how to ensure the war preparedness serviceability rate is solved quickly in the work of the equipment technology supportability under the high tech war.

The maintenance quality evaluation is the important content of the full system and life control and the important measure ensuring the maintenance quality and the equipment reliability ${ }^{[1]}$. The maintenance work is evaluated scientifically and logically through the maintenance quality evaluation. The exact evaluation can improve the maintenance quality and availability of the equipment and prolong the used life of the equipment. Studying the theory of the equipment maintenance quality evaluation and exploring the exact evaluation method can promote the maintenance work and the full life control of the equipment, and can consummate the theory of the equipment maintenance. The evaluation model of equipment maintenance quality based on the neural network BP algorithm is set up and used to evaluate the equipment maintenance quality in the paper.

\section{Neural network BP algorithm and the neural network toolbox of MATLAB}

The neural network BP algorithm is the unidirectional spread and multi-layer feedforward neural network $^{[2]}$, and the network has the connotative nodes of the layer or multilayer beside the in-out nodes, and between the nodes of the layer don't connect, and the interpass nodes connect outright. The import signal sends through all connotative nodes from the import layer to the output layer. The output of the layer only affects the output of the next layer. The transfer function of the nodes is the tanh-regression sigmoid function, and the transfer function of the output nodes is the linearity function. The study process of the neural network BP algorithm makes up of the forward spread and the reverse spread. The states of the nerve cells of the layer only affect the states of the nerve cells of the next layer. There is the error between the practical output value and the expectation output value if the expectation output isn't received, and the error signal returns along the quondam connect way. The error signal spreads to the import layer gradually and puts up calculating through modifying the weights of the nerve cells of the layers. After that the error signal spreads forwardly. The two processes go along time and again, and that makes the error signal least. The neural network BP algorithm has the non linear mapping ability so that it can approach the random non linear function commendably. The neural network BP algorithm with three layers can approach the random continuous function at the random precision. 
The neural network toolbox of MATLAB bases on the neural network theory, and the tool function of the typical neural network with MATLAB language ${ }^{[3]}$. A lot of the toolbox functions about the neural network BP algorithm are written in MATLAB and the strong tools for the research of the neural network BP algorithm. The primary toolbox functions are used as follows: (1) the initialization function: $\operatorname{rands}()$ is used to endue the initial values of the weights and threshold value of the network. (2) the nerve cell transfer function: purelin() is the linearity transfer function and adapted by the nerve cells of the output layers using the BP arithmetic to train; tansig() is the tanh-regression sigmoid transfer function and used to make the import value within the range of "minus infinity" to "infinity" mapped the range of "minus one" to "one"; $\operatorname{logsig}()$ is the pair of sigmoid transfer function and used to make the import value within the range of "minus infinity" to "infinity" mapped the range of "zero" to "one", and that are adapted by the nerve cells of the connotative layers using the BP arithmetic to $\operatorname{train}^{[4]}$. (3) the network training function: trainbpx() is used to set up the neural network $\mathrm{BP}$ algorithm. (4) the network simulation function: simuff() is used to simulate the neural network BP algorithm that has been trained already.

\section{Set up the evaluation model of equipment maintenance quality based on the neural network BP algorithm}

The evaluation model of equipment maintenance quality based on the neural network BP algorithm is set up mainly making use of the toolbox of the neural network BP algorithm of MATLAB. The steps ${ }^{[5]}$ are as follows:

(1) confirming the training swatches. The import swatches and the aim swatches are confirmed, and in others words that the import factors and the output parameters are confirmed. In general, a lot of the example swatches are organized so that the prediction precision the evaluation model of equipment maintenance quality based on the neural network BP algorithm can reach is very high.

(2) setting up and initializing the network. The structure of the neural network is confirmed according to the number of the factors, and in other words that the neural network has the number of the import, the output, the connotative nodes and so on. The number of the import nodes lies on the number of the import factors, and the number of the output nodes lies on the number of the output parameters, and the number of the connotative layers and nodes is chosen by the experience. The transfer function between the import layers, the connotative layers and the output nodes is the nerve cell transfer function of the neural network toolbox of MATLAB. The network is initialized by the initialization function of the toolbox.

(3) training the network. The network is trained by the network training function of the toolbox of the neural network BP algorithm of MATLAB. The training is stopped when the precision is reached, and all weight coefficients are worked out.

(4) evaluating the equipment maintenance quality. That are realized by the feedforward network simulation function of the toolbox of the neural network BP algorithm of MATLAB. The equipment maintenance quality is worked out through imputing the values of the influence factors into the evaluation model of equipment maintenance quality based on the neural network BP algorithm that has been trained well.

\section{The example analysis}

The maintenance qualities of all parts of the artillery and the whole artillery are evaluated by the experts according to the steps in setting up the evaluation model of equipment maintenance quality based on the neural network BP algorithm. The twenty training swatches are received, as shown in table first:

Table.1 Training swatches of the neural network BP algorithm

\begin{tabular}{|c|c|c|c|c|c|c|c|c|c|c|c|c|c|c|c|c|}
\hline number & $\mathrm{P}_{1}$ & $\mathrm{P}_{2}$ & $\mathrm{P}_{3}$ & $\mathrm{P}_{4}$ & $\mathrm{P}_{5}$ & $\mathrm{P}_{6}$ & $\mathrm{P}_{7}$ & $\mathrm{P}_{8}$ & $\mathrm{P}_{9}$ & $\mathrm{P}_{10}$ & $\mathrm{P}_{11}$ & $\mathrm{P}_{12}$ & $\mathrm{P}_{13}$ & $\mathrm{P}_{14}$ & $\mathrm{P}_{15}$ & $\mathrm{P}$ \\
\hline 1 & 6.5 & 7.6 & 8.0 & 7.7 & 7.0 & 8.2 & 8.7 & 8.1 & 8.5 & 7.9 & 9.0 & 6.9 & 8.0 & 8.2 & 7.8 & 7.5 \\
\hline
\end{tabular}




\begin{tabular}{|c|c|c|c|c|c|c|c|c|c|c|c|c|c|c|c|c|}
\hline 2 & 8.3 & 8.1 & 8.5 & 8.6 & 8.0 & 8.8 & 8.6 & 9.0 & 8.2 & 8.5 & 9.2 & 9.3 & 8.3 & 8.1 & 8.6 & 8.5 \\
\hline 3 & 9.2 & 7.2 & 8.8 & 7.6 & 8.2 & 7.9 & 8.3 & 9.1 & 9.0 & 7.9 & 6.9 & 9.5 & 8.5 & 8.6 & 8.7 & 8.0 \\
\hline 4 & 7.5 & 8.3 & 7.8 & 7.8 & 7.5 & 8.6 & 7.4 & 8.5 & 8.0 & 8.3 & 8.8 & 7.8 & 8.8 & 8.9 & 8.9 & 8.0 \\
\hline 5 & 7.8 & 8.5 & 7.2 & 7.5 & 8.0 & 6.9 & 7.8 & 8.6 & 7.0 & 8.6 & 9.3 & 8.8 & 8.7 & 7.9 & 7.9 & 8.0 \\
\hline 6 & 8.0 & 6.8 & 8.3 & 8.5 & 7.0 & 8.9 & 7.5 & 8.4 & 8.9 & 8.8 & 7.9 & 8.5 & 8.2 & 8.1 & 8.4 & 8.3 \\
\hline 7 & 8.5 & 7.0 & 7.5 & 7.8 & 8.8 & 9.2 & 7.9 & 8.0 & 8.6 & 7.9 & 8.6 & 7.6 & 7.9 & 8.6 & 8.5 & 8.1 \\
\hline 8 & 7.0 & 7.7 & 8.0 & 8.5 & 9.0 & 8.8 & 8.5 & 8.9 & 9.2 & 8.5 & 7.9 & 9.0 & 8.9 & 8.4 & 8.8 & 9.0 \\
\hline 9 & 6.0 & 6.8 & 7.5 & 7.0 & 7.8 & 8.0 & 7.8 & 8.3 & 7.5 & 6.9 & 7.7 & 7.9 & 8.5 & 7.9 & 8.6 & 7.5 \\
\hline 10 & 6.8 & 7.0 & 8.0 & 7.5 & 7.6 & 8.2 & 8.6 & 7.9 & 8.3 & 7.7 & 6.9 & 8.7 & 6.8 & 6.5 & 7.6 & 7.5 \\
\hline 11 & 9.3 & 8.9 & 8.6 & 8.5 & 8.3 & 9.1 & 9.2 & 9.6 & 8.6 & 8.9 & 8.9 & 7.9 & 9.1 & 9.4 & 8.9 & 9.0 \\
\hline 12 & 9.5 & 9.0 & 9.2 & 9.0 & 9.6 & 9.3 & 9.0 & 9.8 & 9.2 & 9.3 & 9.6 & 8.9 & 9.5 & 9.5 & 8.8 & 9.3 \\
\hline 13 & 8.8 & 8.6 & 8.5 & 8.0 & 8.6 & 8.5 & 8.9 & 8.9 & 8.1 & 8.6 & 8.8 & 8.3 & 8.9 & 9.0 & 8.6 & 8.5 \\
\hline 14 & 8.3 & 8.0 & 8.7 & 8.5 & 8.0 & 8.6 & 8.3 & 8.8 & 8.4 & 8.7 & 9.0 & 8.2 & 8.5 & 9.1 & 8.4 & 8.5 \\
\hline 15 & 6.0 & 6.5 & 7.0 & 6.8 & 7.5 & 7.0 & 7.6 & 7.5 & 7.9 & 7.8 & 8.0 & 7.6 & 8.6 & 8.2 & 7.9 & 7.0 \\
\hline 16 & 8.0 & 7.9 & 7.3 & 7.2 & 7.5 & 8.1 & 8.5 & 7.6 & 8.6 & 8.9 & 9.0 & 7.3 & 7.8 & 7.9 & 8.2 & 7.6 \\
\hline 17 & 8.6 & 8.0 & 8.5 & 8.6 & 8.0 & 8.8 & 8.3 & 8.2 & 8.4 & 8.8 & 9.0 & 8.5 & 8.1 & 9.0 & 9.1 & 8.7 \\
\hline 18 & 9.6 & 8.6 & 9.2 & 8.0 & 8.9 & 9.5 & 9.5 & 9.1 & 9.4 & 9.6 & 8.8 & 8.6 & 8.4 & 9.2 & 9.3 & 9.2 \\
\hline 19 & 7.9 & 8.2 & 8.5 & 8.0 & 7.9 & 7.8 & 8.6 & 8.4 & 8.2 & 8.3 & 8.9 & 8.9 & 8.6 & 8.5 & 8.2 & 8.0 \\
\hline 20 & 8.8 & 7.3 & 8.5 & 9.0 & 8.2 & 7.9 & 8.0 & 9.2 & 8.6 & 8.0 & 7.9 & 9.1 & 8.5 & 8.2 & 9.4 & 8.5 \\
\hline
\end{tabular}

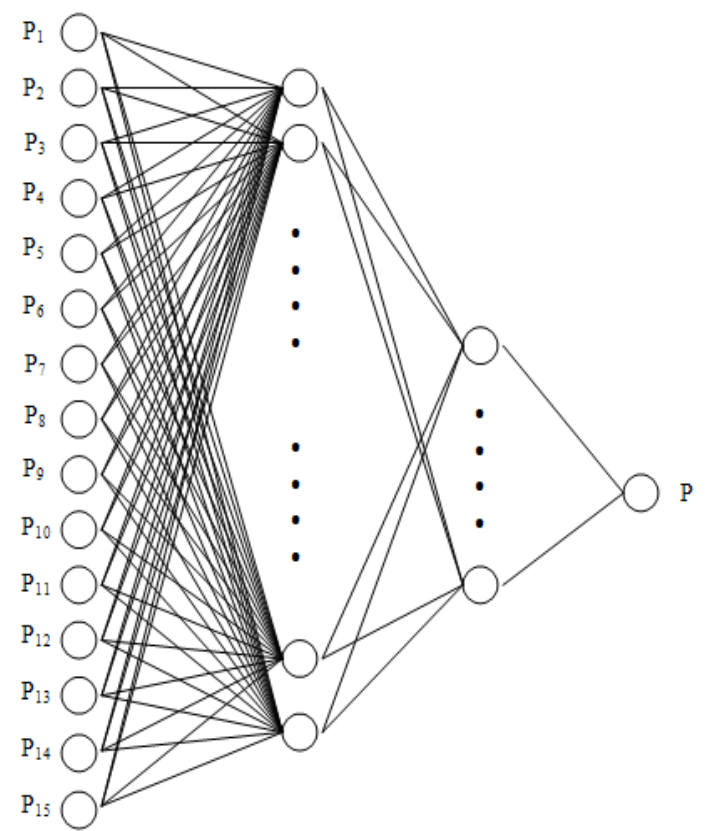

Fig.1 Evaluation model based on the neural network BP algorithm

The data of the table first is transformed to twenty pairs of the training swatches of the evaluation model of equipment maintenance quality based on the neural network BP algorithm, and the model is trained by twenty training swatches, and the training is stopped after that the precision is reached. The import layer has fifteen nodes, and there are two connotative layers, and the first connotative layer has 
five hundred nodes, and the twice connotative layer has three hundred nodes, and the output has one node.

The two pairs of the training swatches are inputted the network for testing the effect of the network training, and the evaluation results are calculated as shown in table twice. The predictable value is close to the practical value, and but there is the certain error between them because the number of the swatches is small, and the error becomes little when the number of the swatches is large.

Table. 2 comparing the prediction value and the measure value

\begin{tabular}{|c|c|c|c|c|c|c|c|c|c|c|c|c|c|c|c|c|c|}
\hline number & $\mathrm{P}_{1}$ & $\mathrm{P}_{2}$ & $\mathrm{P}_{3}$ & $\mathrm{P}_{4}$ & $\mathrm{P}_{5}$ & $\mathrm{P}_{6}$ & $\mathrm{P}_{7}$ & $\mathrm{P}_{8}$ & $\mathrm{P}_{9}$ & $\mathrm{P}_{10}$ & $\mathrm{P}_{11}$ & $\mathrm{P}_{12}$ & $\mathrm{P}_{13}$ & $\mathrm{P}_{14}$ & $\mathrm{P}_{15}$ & $\mathrm{P}_{\mathrm{Y}}$ & $\mathrm{P}_{\mathrm{S}}$ \\
\hline 1 & 7.1 & 8.3 & 9.2 & 9.4 & 8.5 & 8.8 & 7.9 & 8.6 & 7.6 & 9.0 & 7.5 & 8.7 & 8.1 & 8.0 & 9.3 & 8.2 & 8.3 \\
\hline 2 & 6.5 & 7.2 & 7.8 & 8.2 & 8.5 & 8.7 & 8.1 & 9.0 & 7.9 & 8.3 & 8.8 & 8.4 & 9.2 & 9.6 & 8.9 & 8.1 & 8.0 \\
\hline
\end{tabular}

\section{Conclusion}

The neural network BP algorithm has the capabilities of self-organizing, self-learning and nonlinear approximation, and the approximation of function using the neural network is applied widely. The equipment maintenance quality is predicted using the neural network BP algorithm, and the precision of the network is high according to the simulation results, and especially the neural network BP algorithm can solve the high nonlinear problem and overcome the deficiency of the traditional method. It is a new method to predict the equipment maintenance quality.

\section{References}

[1] Yang Wei-min, Ruan Lian, Yu Zhao etc. Reliability·Maintainability·indemnificatory pandect [M]. Beijing: National Defence Industry Press, 1995.

[2] Jiang Zong-li. Introduction to Artificial Neural Network[M]. Beijing:Higher Education Press, 2001.

[3] Lou Shun-tian, Shi Yang. Systems Analysis and Design - the Nerve Network[M]. Xian: Xian Electronics Science and Technology University Press, 1998.

[4] Ma Rui. Artificial Neural Network Theory [M]. Beijing:Machine Industry Press, 2010.

[5] Wen Xin, Zhou Lu, Wang Dan-li etc. the Application and Design of the Nerve Network basing on MATLAB[M]. Beijing: Science Press, 2000. 